\title{
Glicerol en el comportamiento productivo y características de la canal y carne de cabras: un metaanálisis
}

\author{
Glycerol on the productive performance and characteristics of the carcass and \\ meat of goat: a meta-analysis
}

\author{
Gustavo Daniel Vega Britez ${ }^{1,5}$, Fernando Miranda de Vargas Junior ${ }^{2}$, \\ Marciana Retore ${ }^{3}$, Ariadne Patricia Leonardo ${ }^{2}$, Nelson David Lesmo Duarte ${ }^{1}$, \\ José Augusto Velazquez Duarte ${ }^{1}$, Jorge Darío Alvarenga Serafini', \\ Modesto Osmar da Silva Oviedo ${ }^{4}$
}

\section{Resumen}

\begin{abstract}
El objetivo del presente estudio meta-analítico fue evaluar el efecto del glicerol en la dieta sobre el comportamiento productivo, digestibilidad de nutrientes y las características de la canal y carne de cabras en engorde. Se hizo una revisión sistemática utilizando las bases de datos de Google Scholar, ScienceDirect y SciELO. Palabras clave como crude glycerin, intake, goat, kids, carcass, meat quality fueron utilizados. Como criterios de inclusión de los artículos científicos se consideraron la presencia de un tratamiento testigo ( $0 \%$ de glicerol) y por lo menos un porcentaje de glicerol en la dieta, datos referentes a la digestibilidad de los nutrientes, respuesta productiva, características de la canal y carne y nivel de glicerol en la glicerina bruta en trabajos publicados entre 2014 y 2019. Se seleccionaron 56 artículos, de los cuales 11 reunieron los criterios de inclusión.
\end{abstract}

${ }^{1}$ Facultad de Ciencias Agrarias, Universidad Nacional de Asunción, filial Pedro Juan Caballero. Orientación Producción Animal, Pedro Juan Caballero, Paraguay

${ }^{2}$ Faculdade de Ciências Agrárias, Universidade Federal da Grande Dourados, Departamento de Zootecnia, Dourados MS, Brasil

${ }^{3}$ Embrapa Agropecuária Oeste, Manejo Animal e Transferência de Tecnologia, Dourados MS, Brasil

${ }^{4}$ Facultad de Ciencias Agrarias, Universidad Nacional de Concepción, Departamento de Producción Agrícola, Concepción, Paraguay

${ }^{5}$ E-mail: gda.vega@gmail.com; https://orcid.org/0000-0002-4051-2110

Recibido: 22 de julio de 2020

Aceptado para publicación: 31 de marzo de 2021

Publicado: 23 de junio de 2021

CLos autores. Este artículo es publicado por la Rev Inv Vet Perú de la Facultad de Medicina Veterinaria, Universidad Nacional Mayor de San Marcos. Este es un artículo de acceso abierto, distribuido bajo los términos de la licencia Creative Commons Atribución 4.0 Internacional (CC BY 4.0) [https:// creativecommons.org/licenses/by/4.0/deed.es] que permite el uso, distribución y reproducción en cualquier medio, siempre que la obra original sea debidamente citada de su fuente original 
Ante la heterogeneidad o amplitud de los niveles de glicerol, los niveles fueron agrupados: $0,1.27-4.38$ y $6.40-9.60 \%$ de glicerol en base a la materia seca de la dieta. Análisis de normalidad, variancia y de regresión fueron realizadas a partir de los datos de cada variable dentro de cada nivel de glicerol en la dieta. Se evidenció la alta variación de los niveles de glicerol en glicerina bruta. Glicerol hasta $9.60 \%$ en la dieta no compromete la digestibilidad, consumo, principales características de la canal y carne de cabra.

Palabras clave: alimentación alternativa, agroindustrias, calidad de la carne, rumiantes, subproductos

\section{Abstract}

The aim of the present meta-analytical study was to evaluate the effect of glycerol in the diet on the productive performance, nutrient digestibility and the characteristics of the carcass and meat of fattening goats. A systematic review was made using the Google Scholar, ScienceDirect and SciELO databases. Keywords such as crude glycerine, intake, goat, kids, carcass, meat quality were used. As inclusion criteria of the scientific articles, the presence of a control treatment ( $0 \%$ glycerol) and at least a percentage of glycerol in the diet, data regarding the digestibility of nutrients, productive response, characteristics of the carcass and meat and glycerol level in crude glycerin were considered in papers published between 2014 and 2019. In total, 56 articles were selected, of which 11 met the inclusion criteria. Given the heterogeneity or amplitude of the glycerol levels, the levels were grouped: $0,1.27-4.38$ and $6.40-9.60 \%$ of glycerol based on the dry matter of the diet. Normality, variance and regression analyses were performed from the data of each variable within each level of glycerol in the diet. The high variation of glycerol levels in crude glycerine was evidenced. Glycerol up to $9.60 \%$ in the diet does not compromise digestibility, consumption, main characteristics of the carcass and goat meat.

Key words: alternative feeding, agro-industries, by-products, meat quality, ruminants

\section{INTRODUCCIÓN}

La preocupación por un ambiente más saludable, la necesidad de la renovación progresiva de la matriz energética y el gran potencial agrícola a nivel regional, especialmente en Brasil (Lei N. $.^{\circ} 11097 / 2005$ ) y Paraguay (Ley N. $\left.{ }^{\circ} 2748 / 2005\right)$ se reglamentó la adición gradual de combustibles de fuentes renovables en el combustible fósil, originándose el biodiesel, lo cual propició el aumento de su producción a partir de cultivos agrícolas como la soja y el maíz. Este hecho generó inevitablemente una gran cantidad de subproductos, entre ella la glicerina bruta, la cual se estima que una tonelada de biodiesel producida genera $100 \mathrm{~kg}$ glicerina bruta (ANP, 2019).

Son diversas las utilidades de la glicerina bruta, dependiendo del nivel de purificación, y se viene explorando su uso en el sector agropecuario. La glicerina mejora la composición química del ensilado de pastos tropicales (Machado et al., 2019) y presenta gran potencial como fuente energética en la dieta de animales de producción como cerdos (Pinto et al., 2019), peces (Theisen et al., 2019), pollos de carne (Silva et al., 2019), bovino lechero (Paiva et al., 2016) y de carne (Castagnino et al., 2018), ovinos (Valença et al., 2020) y cabras (Matos et al., 2018). 
La adición de glicerina bruta en la dieta permite el reemplazo gradual de parte de los granos energéticos que componen la dieta, como maíz y sorgo, sin comprometer la calidad del producto final, sea carne o leche (Alves e Dias et al., 2018). Según Avila et al. (2011), el glicerol tiene el potencial de modificar las características de fermentación hacia la vía glucogénica y mejorar la digestibilidad de la dieta en pequeños rumiantes.

Inclusión de glicerina bruta en la dieta de cabras no afecta la digestibilidad de los nutrientes (Chanjula et al., 2014) ni el desempeño, canal y calidad de carne, siendo posible substituir al maíz hasta el $20 \%$ de la materia seca de la dieta (Chanjula et al., 2015). Sin embargo, existen discrepancias entre los hallazgos publicados en la literatura científica, principalmente debido al nivel y pureza del glicerol y la etapa de producción de los animales (Kholif, 2019). Tal es así que glicerina bruta de baja pureza no se recomienda para reemplazar al maíz en la dieta de cabras porque reduce el consumo y la digestibilidad de varias fracciones nutricionales y disminuye el rendimiento de la canal (Bezerra et al., 2019), pero glicerina bruta de $91.62 \%$ de pureza (glicerol) no afecta las características de la carcasa (Matos et al., 2018). Asimismo, AbuGhazaleh et al. (2011) indican que glicerina bruta de alta pureza permite sustituir hasta el 15\% del maíz sin presentar efectos adversos sobre la fermentación, la digestión o las bacterias ruminales.

Las discrepancias existentes sobre el uso de glicerol en la alimentación animal y la poca atención a la concentración de glicerol que compone la glicerina bruta sobre el comportamiento productivo y las características de la canal y de la carne de la cabra, dificulta realizar comparaciones válidas entre estudios. Ante esto, el objetivo del presente estudio fue evaluar el efecto del glicerol en la dieta sobre el desempeño productivo y características de la canal y carne de cabras mediante un metaanálisis.

\section{Materiales y Métodos}

Se realizó una búsqueda de trabajos sobre comportamiento productivo, características de la canal y carne de cabras con inclusión de glicerina bruta en la dieta, mediante una revisión sistemática, utilizando las bases de datos de Google Scholar, ScienceDirect y SciELO. La búsqueda y selección de los artículos científicos se realizó entre enero y mayo de 2020, utilizando las palabras clave crude glycerin, intake, goat, kids, carcass y meat quality. Como criterio de inclusión se consideraron la presencia de un tratamiento control ( $0 \%$ de glicerina bruta - GB) y por lo menos un porcentaje de GB en la dieta de animales a término o engorde, sea en confinamiento o en pastura, presencia de datos referentes a digestibilidad de los nutrientes (materia seca, proteína bruta, extracto etéreo y fibra detergente neutro), comportamiento animal (peso corporal inicial [PCI] y final [PCFi], consumo de materia seca [CMS], ganancia promedio diaria de peso [GPD] y conversión alimenticia [CA]), características de la canal (peso de la canal caliente y fría, rendimiento de la canal fría y área de ojo de lomo), cortes comerciales y características fisicoquímicas de la carne (pierna, lomo, paleta, $\mathrm{pH}$, fuerza de cizallamiento, humedad y mineral) y nivel de glicerol en la glicerina bruta. Se consideraron los artículos publicados entre 2014 y 2020 (Cuadro 1), según la metodología de Moher et al. (2009), que comprende la identificación, selección y evaluación, elegibilidad y la inclusión (Figura 1).

El resultado de la búsqueda inicial identificó 58 artículos científicos, y luego de la revisión del título se descartaron 36 artículos por no considerar a las cabras como sujeto del estudio. Luego de la revisión del resumen de los 22 artículos seleccionados se descartaron ocho por no considerar la calidad de la leche y el consumo. Así, 14 artículos presentaron las condiciones requeridas para su análisis; sin embargo, al leer la metodología se excluyeron tres artículos por no mencionar el 


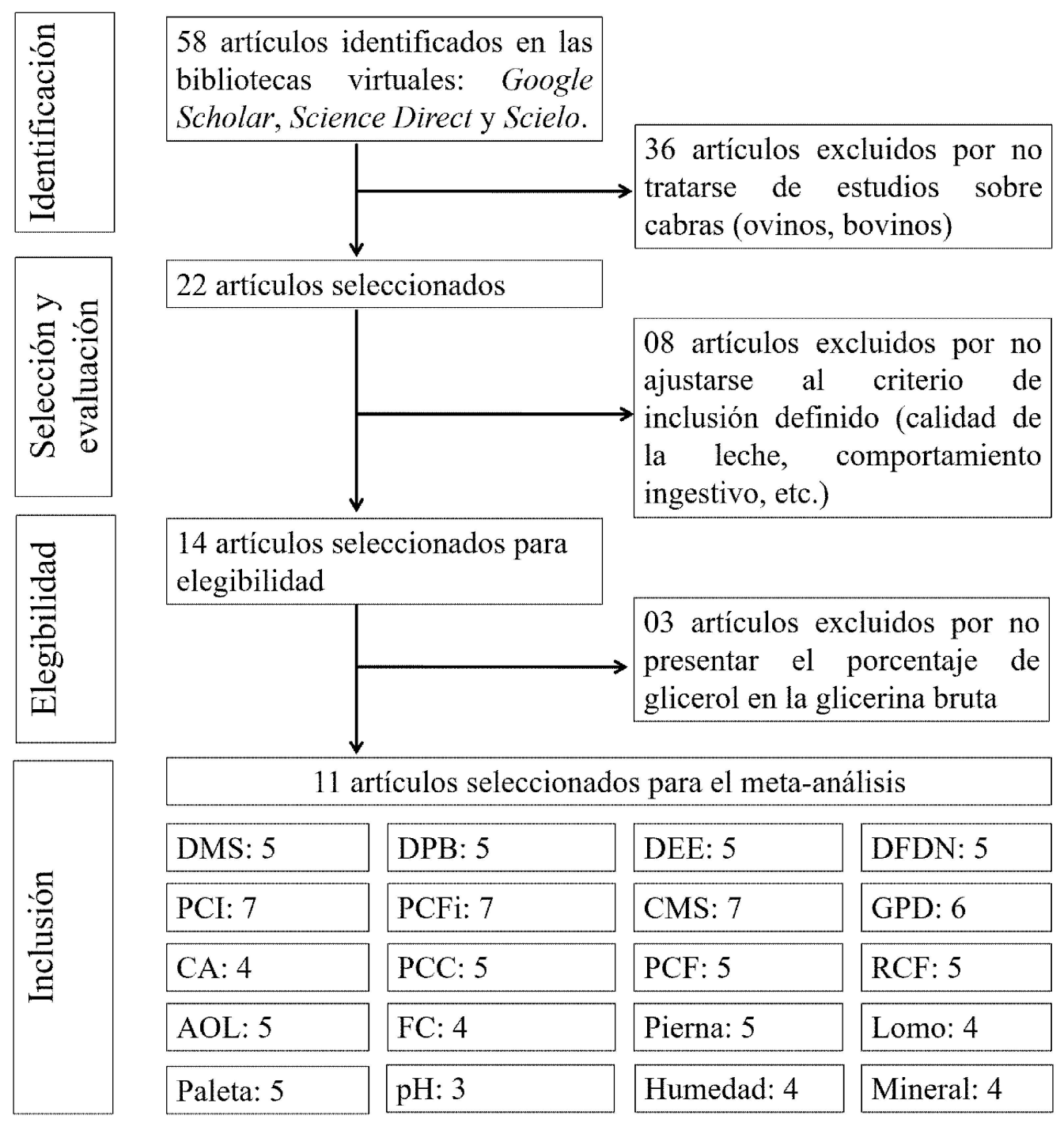

Figura 1. Diagrama de flujo PRISMA (adaptado de Moher et al., 2009). DMS=digestibilidad de la materia seca; $D P B=$ digestibilidad de la proteina bruta; $D E E=$ digestibilidad de extracto etéreo; $D F D N=$ digestibilidad de la fibra detergente neutro; $P C I=$ peso corporal inicial; $C M S=$ consumo de materia seca; $C A=$ conversión alimenticia; $P C F i=$ peso corporal final; $G P D=$ ganancia promedio diaria de peso; $P C C=$ peso de la canal caliente; $P C F=$ peso de la canal fría; $R C F=$ rendimiento de la canal fría; $A O L=$ área de ojo de lomo; $F C=$ fuerza de cizallamiento

porcentaje de glicerol en la glicerina bruta. Al final, los datos para los análisis se tomaron de 11 artículos que reunieron los criterios de inclusión para el metaanálisis (Cuadro 1).
Los datos de las variables que corresponden a glicerina bruta fueron obtenidos directamente de los artículos seleccionados. Los datos para glicerol fueron obtenidos trans- 
Cuadro 1. Descripción del estudio primario incluido en el metaanálisis $(\mathrm{n}=11)$

\begin{tabular}{|c|c|c|c|c|}
\hline Autores & País & $\begin{array}{l}\text { Sistema de } \\
\text { terminación }\end{array}$ & $\begin{array}{l}\text { Glicerol } \\
(\%)\end{array}$ & Variables \\
\hline $\begin{array}{l}\text { Alves e Dias et } \\
\text { al. (2018) }\end{array}$ & Brasil & Confinamiento & 89.10 & $\begin{array}{l}\text { PCI, PCFi, CMS, PCC, } \\
\text { PCF, RCF, AOL, FC, } \\
\text { Pierna, Lomo, Paleta, pH }\end{array}$ \\
\hline $\begin{array}{l}\text { Bezerra et al. } \\
\text { (2019) }\end{array}$ & Brasil & Confinamiento & 43.40 & $\begin{array}{l}\text { DMS, DPB, DEE, DFDN, } \\
\text { PCI, PCFi, GPD, CA }\end{array}$ \\
\hline $\begin{array}{l}\text { Chanjula et al. } \\
\text { (2014) }\end{array}$ & Tailandia & Confinamiento & 87.61 & $\begin{array}{l}\text { DMS, DPB, DEE, DFDN, } \\
\text { CMS }\end{array}$ \\
\hline $\begin{array}{l}\text { Chanjula et al. } \\
(2015)\end{array}$ & Tailandia & Confinamiento & 87.61 & $\begin{array}{l}\text { PCI, PCFi, CMS, GPD, CA, } \\
\text { PCC, PCF, RCF, AOL, FC, } \\
\text { Pierna, Lomo, Paleta, } \\
\text { Humedad, Mineral }\end{array}$ \\
\hline $\begin{array}{l}\text { Chanjula et al. } \\
\text { (2016) }\end{array}$ & Tailandia & Confinamiento & 63.42 & $\begin{array}{l}\text { DMS, DPB, DEE, DFDN, } \\
\text { CMS }\end{array}$ \\
\hline $\begin{array}{l}\text { Chanjula et al. } \\
\text { (2018) }\end{array}$ & Tailandia & Confinamiento & 63.41 & $\begin{array}{l}\text { PCI, PCFi, CMS, GPD, CA, } \\
\text { PCC, PCF, RCF, AOL, FC, } \\
\text { Pierna, Lomo, Paleta, pH, } \\
\text { Humedad, Mineral }\end{array}$ \\
\hline $\begin{array}{l}\text { Dias et al. } \\
\text { (2016) }\end{array}$ & Brasil & Confinamiento & 87.00 & $\begin{array}{l}\text { PCI, PCFi, CMS, GPD, } \\
\text { PCC, PCF, RCF, AOL, } \\
\text { Pierna, Paleta }\end{array}$ \\
\hline $\begin{array}{l}\text { Gomes et al. } \\
\text { (2020a) }\end{array}$ & Brasil & Confinamiento & 63.06 & $\begin{array}{l}\text { DMS, DPB, DEE, DFDN, } \\
\text { PCI, PCFi, CMS, GPD, CA }\end{array}$ \\
\hline $\begin{array}{l}\text { Matos et al. } \\
\text { (2018) }\end{array}$ & Brasil & Confinamiento & 91.62 & $\begin{array}{l}\text { PCC, PCF, RCF, AOL, FC, } \\
\text { Pierna, Lomo, Paleta, pH }\end{array}$ \\
\hline $\begin{array}{l}\text { Rocha et al. } \\
(2015)\end{array}$ & Brasil & Confinamiento & 80.00 & Humedad, Mineral \\
\hline $\begin{array}{l}\text { Santos et al. } \\
(2015)\end{array}$ & Brasil & Confinamiento & 80.02 & $\begin{array}{l}\text { DMS, DPB, DEE, DFDN, } \\
\text { PCI, PCFi, GPD }\end{array}$ \\
\hline \multicolumn{2}{|c|}{ Mínimo } & & 43.40 & \\
\hline \multicolumn{2}{|c|}{ Media } & & 76.02 & \\
\hline \multicolumn{2}{|c|}{ Máximo } & & 91.62 & \\
\hline \multicolumn{2}{|c|}{ CV $(\%)$} & & 20.31 & \\
\hline
\end{tabular}

$\mathrm{DMS}=$ digestibilidad de la materia seca; $\mathrm{DPB}=$ digestibilidad de la proteína bruta; $\mathrm{DEE}=$ digestibilidad de extracto etéreo; $\mathrm{DFDN}=$ digestibilidad de la fibra detergente neutro; $\mathrm{PCl}=$ peso corporal inicial; $\mathrm{CMS}=$ consumo de materia seca; $\mathrm{CA}=$ conversión alimenticia; $\mathrm{PCF}=$ peso corporal final; $\mathrm{GPD}=$ ganancia promedio diaria de peso; $\mathrm{PCC}=$ peso de la canal caliente; $\mathrm{PCF}=$ peso de la canal fría; $\mathrm{RCF}=$ rendimiento de la canal fría; $\mathrm{AOL}=$ área de ojo de lomo; $\mathrm{FC}=$ fuerza de cizallamiento; CV: coeficiente de variación 
formando los datos presentados en los artículos (porcentaje de glicerina bruta en la MS por porcentaje de glicerol informado en cada artículo), a fin de formar tratamientos con diferentes niveles de glicerol en la MS de la dieta. Ante la heterogeneidad o amplitud de los niveles utilizados en las publicaciones, los niveles de glicerol fueron agrupados como sigue: $0,1.27-4.38$. $6.40-9.60 \%$. Dos trabajos consideraron niveles de glicerol mayores a $9.60 \%$ en la MS, pero no fueron considerados para el metaanálisis.

Se utilizó el análisis de normalidad (test de Shapiro-Wilk), variancia y de regresión lineal a partir de datos de cada variable dentro de cada nivel de glicerol en la dieta. Se empleó el software estadístico AgroEstat $₫$ para diferencias al nivel de $\mathrm{p}<0.05$.

\section{Resultados y Discusión}

La prueba de Shapiro-Wilk evidenció una distribución normal de los datos. El número de animales por variable evaluadas se detallan en el Cuadro 2. Para las variables relacionadas al comportamiento productivo se identificó un mayor número de animales, seguido para las variables relacionadas a digestibilidad de los nutrientes, características de la canal y cortes comercial y calidad de la carne. La diferencia en el número de animales por variable se debe a que no todos los artículos seleccionados evaluaron las mismas variables; incluso, algunos solo evaluaron un corte comercial como la pierna.

Los niveles de glicerol no influenciaron $(p>0.05)$ la respuesta productiva de las cabras, tales como el PCI, PCFi, CMS, GPD y CA (Cuadro 3). Niveles de glicerol hasta $9.60 \%$ en base a la MS de la dieta no causa efecto deletéreo sobre el desempeño animal.

Chanjula et al. (2015) consideran viable el uso de glicerina bruta, correspondiente a $8.76 \%$ de glicerol en ovinos, mientras que Chanjula et al. (2018) consideran hasta 3.81\% de glicerol en la MS en cabras en terminación, siendo al parecer la estrategia más interesante, ya que promueve el mayor rendimiento de los animales. Sin embargo, Chanjula (2018) en una revisión sistemática constata que la glicerina bruta puede ser utilizada hasta $20 \%$ de la MS como fuente de energía; no obstante, esta conclusión es relativa, debido a que no informan el nivel de pureza o porcentaje de glicerol presente en la glicerina bruta.

Al evaluar el nivel de glicerol en la dieta sobre la digestibilidad de los nutrientes en el presente metaanálisis fue posible determinar que, hasta los niveles evaluados, el glicerol no afecta la digestibilidad de los nutrientes como materia seca, proteína, extracto etéreo y fibra detergente neutro (Cuadro 4); sin embargo, al evaluar el nivel de glicerina bruta en la dieta, Bezerra et al. (2019) verificaron que $15 \%$ de glicerina bruta en la dieta (MS) perjudica el consumo, la digestibilidad y el rendimiento, lo cual probablemente estaría relacionado con la concentración de glicerol en la glicerina bruta. Estos autores trabajaron con $43.40 \%$ de pureza; es decir, $15 \%$ de glicerina bruta representa $6.51 \%$ de glicerol en la dieta. Allí radica la importancia del nivel de glicerol, quedando demostrado que una baja pureza puede ser perjudicial para los animales en engorde.

En ese sentido, según Barros et al. (2015), la baja pureza (glicerina bruta conteniendo $43.6 \%$ de glicerol y $6 \%$ de metanol) limita el consumo de MS y, consecuentemente, de los demás nutrientes; sin embargo, en el caso de ovinos no afecta el comportamiento productivo y características de la canal, pudiendo ser incluida en hasta $10.84 \%$ (4.72\% de glicerol) en la MS de raciones para ovinos en confinamiento, considerando que niveles de glicerol, metanol y extracto etéreo presentes en glicerina bruta de baja pureza en la dieta pueden contribuir de forma aislada o en conjunto para influenciar el CMS de los animales. Strada et al. (2015) verificaron que la glicerina de baja pureza pude ser incluida en 
Cuadro 2. Número de repeticiones de cabras para las variables evaluadas según los diferentes niveles de glicerol en la dieta

\begin{tabular}{|c|c|c|c|c|}
\hline \multirow{2}{*}{ Variables } & \multicolumn{3}{|c|}{ Niveles de glicerol en la dieta } & \multirow{2}{*}{ Total } \\
\hline & $0 \%$ & $1.27-4.38 \%$ & $6.40-9.60 \%$ & \\
\hline \multicolumn{5}{|c|}{ Desempeño animal } \\
\hline PCI (kg) & 49 & 62 & 48 & 159 \\
\hline PCFi (kg) & 49 & 62 & 48 & 159 \\
\hline CMS (kg/día) & 58 & 79 & 48 & 185 \\
\hline GPD (kg/día) & 42 & 62 & 41 & 145 \\
\hline $\mathrm{CA}(\mathrm{g} / \mathrm{kg})$ & 32 & 52 & 26 & 110 \\
\hline \multicolumn{5}{|c|}{ Digestibilidad de nutrientes (\%) } \\
\hline Materia seca & 37 & 43 & 28 & 108 \\
\hline Proteína bruta & 37 & 43 & 28 & 108 \\
\hline Extracto etéreo & 37 & 43 & 28 & 108 \\
\hline Fibra detergente & 37 & 43 & 28 & 108 \\
\hline \multicolumn{5}{|c|}{ Características de la canal } \\
\hline PCC (kg) & 33 & 31 & 27 & 91 \\
\hline PCF (kg) & 33 & 31 & 27 & 91 \\
\hline $\mathrm{RCF}(\%)$ & 38 & 31 & 32 & 101 \\
\hline $\mathrm{AOL}\left(\mathrm{cm}^{2}\right)$ & 33 & 31 & 41 & 105 \\
\hline \multicolumn{5}{|c|}{ Cartes comercial y calidad de la carne } \\
\hline Pierna (kg) & 33 & 31 & 27 & 91 \\
\hline Lomo (kg) & 28 & 26 & 22 & 76 \\
\hline Paleta $(\mathrm{kg})$ & 33 & 31 & 27 & 91 \\
\hline $\mathrm{pH}(24 \mathrm{~h})$ & 20 & 18 & 28 & 66 \\
\hline $\mathrm{FC}\left(\mathrm{kgf} / \mathrm{cm}^{2}\right)$ & 28 & 26 & 36 & 90 \\
\hline Humedad (\%) & 19 & 31 & 18 & 68 \\
\hline Mineral (\%) & 19 & 31 & 18 & 68 \\
\hline
\end{tabular}

la dieta de bovinos no castrados en terminación a pasto con mejoría en el comportamiento productivo y aumento de los beneficios económicos; asimismo, Gomes et al. (2020b) indicaron que se puede incluir hasta $11.34 \%$ en la dieta sin influir negativamente en el peso y el rendimiento de los órganos y vísceras.
Schröder y Südekum (2007) clasificaron la glicerina bruta en baja (63.3\%), media $(85.3 \%)$ y alta $(99.8 \%)$ pureza de glicerol. Sin embargo, independientemente de la pureza, se puede incluir hasta $10 \%$ en la materia seca de la dieta de rumiantes sin afectar negativamente el ambiente ruminal y la 
Cuadro 3. Valores promedios del comportamiento productivo de cabras con diferentes niveles de inclusión de glicerol en la dieta en base a la materia seca

\begin{tabular}{|c|c|c|c|c|c|c|}
\hline \multirow{2}{*}{ Variables } & \multicolumn{3}{|c|}{ Niveles de glicerol (\% MS) } & \multirow{2}{*}{$\begin{array}{l}\text { CV } \\
(\%)\end{array}$} & \multicolumn{2}{|c|}{ valor $\mathrm{p}$} \\
\hline & 0 & $1.27-4.38$ & $6.40-9.60$ & & Gli & $\mathrm{L}$ \\
\hline PCI $(\mathrm{kg})^{1}$ & 18.51 & 18.37 & 18.63 & 14.16 & - & - \\
\hline PCFi $(k g)^{1}$ & 27.37 & 26.83 & 26.09 & 14.55 & 0.636 & 0.550 \\
\hline CMS $(\mathrm{kg} / \mathrm{d})^{1}$ & 0.955 & 0.998 & 0.821 & 24.64 & 0.360 & 0.426 \\
\hline $\operatorname{GPD}(\mathrm{kg} / \mathrm{d})^{2}$ & 0.116 & 0.114 & 0.109 & 22.12 & 0.751 & 0.640 \\
\hline $\mathrm{CA}(\mathrm{g} / \mathrm{kg})^{3}$ & 0.142 & 0.149 & 0.143 & 19.18 & 0.738 & 0.974 \\
\hline
\end{tabular}

$\mathrm{MS}=$ materia seca: $\mathrm{PCl}=$ peso corporal inicial; $\mathrm{PCFi}=$ peso corporal final; $\mathrm{CMS}=$ consumo de materia seca; $\mathrm{GPD}=$ ganancia promedio diario de peso; $\mathrm{CA}=$ conversión alimenticia; $\mathrm{CV}=$ coeficiente de variación

Gli= $0 \%$ de glicerol vs. $1.27-9.60 \%$ de glicerol en la dieta

$\mathrm{L}=$ regresión lineal

${ }^{1}$ Siete artículos incluidos; ${ }^{2}$ Seis artículos incluidos; ${ }^{3}$ Cuatro artículos incluidos

Cuadro 4. Valores promedios (\%) de la digestibilidad de nutrientes con diferentes niveles de inclusión de glicerol en la dieta de cabras en base a la materia seca

\begin{tabular}{lcccccc}
\hline \multirow{2}{*}{ Variables $^{1}$} & \multicolumn{3}{c}{ Niveles de glicerol (\% MS) } & \multirow{2}{*}{ CV $(\%)$} & \multicolumn{2}{c}{ valor $\mathrm{p}$} \\
\cline { 2 - 4 } \cline { 6 - 7 } \cline { 6 - 7 } & 0 & $1.27-4.38$ & $6.40-9.60$ & & Gli & $\mathrm{L}$ \\
\hline DMS (\%) & 73.06 & 73.08 & 75.01 & 6.14 & 0.778 & 0.513 \\
DPB (\%) & 73.26 & 74.11 & 75.25 & 6.35 & 0.634 & 0.546 \\
DEE (\%) & 70.22 & 73.64 & 74.00 & 17.69 & 0.391 & 0.555 \\
DFDN (\%) & 64.22 & 64.41 & 65.16 & 14.67 & 0.930 & 0.885 \\
\hline
\end{tabular}

$\mathrm{DMS}=$ digestibilidad materia seca; $\mathrm{DPB}=$ digestibilidad proteína bruta; $\mathrm{DEE}=$ digestibilidad extracto etéreo; $\mathrm{DFDN}=$ digestibilidad fibra detergente neutro; $\mathrm{CV}=$ coeficiente de variación Gli $=0 \%$ de glicerol vs. 1.27 a $9.60 \%$ de glicerol en la dieta $\mathrm{L}=$ regresión lineal

${ }^{1}$ Cinco artículos incluidos 
digestibilidad de los componentes de la materia orgánica, mientras que glicerol de alta pureza puede incluirse hasta el $20 \%$ en la MS sin afectar la digestibilidad de los nutrientes, inclusive, en cabras lecheras (Novais-Eiras et al., 2018). Así mismo, se ha constatado que la inclusión de glicerol de hasta $2.14 \%$ en la MS no influye sobre los parámetros fisiológicos ni comportamental de cabras (Araújo et al., 2019), hasta 17.5\% de glicerol no perjudica la absorción y retención de nitrógeno (Chanjula et al., 2014) y hasta $8.70 \%$ de glicerol en la dieta no compromete el CMS, la CA y el comportamiento productivo (Dias et al., 2016).

Los resultados del metaanálisis demuestran que hasta $9.60 \%$ de glicerol en la dieta no influye negativamente sobre las características de la canal de cabras (Cuadro 5), pudiendo, por lo tanto, recomendarse su uso como componente energético de la dieta. No obstante, Alves e Dias et al. (2018) evaluaron un nivel de glicerol de $8.63 \%$ en la MS encontrando que el glicerol reduce la ganancia promedio diario de peso, peso corporal final y afecta la conversión alimenticia; sin embargo, Matos et al. (2018) verificaron que la inclusión de hasta $9.16 \%$ en base a la MS de la dieta no reduce las concentraciones plasmáticas de los indicadores de estrés, no altera las características de la canal y no resulta en mayores concentraciones de metales pesados en la carne; asimismo, hasta $8.70 \%$ no afecta el peso de la canal (Dias et al., 2016).

En corderos en engorde, Almeida et al. (2017) recomiendan, dependiendo del precio del maíz, la inclusión de hasta $30 \%$ de glicerina bruta en la dieta (MS), equivalente a $24.90 \%$ ( $83 \%$ pureza) de glicerol, sin causar efectos negativos sobre el consumo, características de la canal y canal, aunque puede obligar a más días de alimentación en el confinamiento. Así mismo, en bovinos en engorde, van Cleef et al. (2017) indican que la inclusión de glicerina bruta hasta $30 \%$ en base a la MS, correspondiente a $25.8 \%(86 \%$ pu- reza) de glicerol tiene el potencial de cambiar la fermentación ruminal, además de mejorar los atributos sensoriales y de la calidad de los ácidos grasos de la carne.

Los cortes comerciales y la calidad de la carne no fueron influenciados $(\mathrm{p}>0.05)$ por la inclusión de glicerol hasta $9.60 \%$ en la dieta (Cuadro 6). No obstante, Alves e Dias et al. (2018) indican que niveles de $8.63 \%$ de glicerol en la MS provoca disminución de los cortes comerciales como la pierna, lomo y costillas; sin embargo, Rocha et al. (2015) verificaron que hasta $9.60 \%$ de glicerol en la MS no afecta la composición química de la carne, excepto para mineral, que presenta un efecto lineal, disminuyendo su concentración al aumentar la inclusión de glicerol en la dieta. En ese sentido, Oliveira et al. (2020) recomiendan ajustar la composición mineral de las dietas en corderos en engorde alimentados con $10 \%$ glicerina bruta $(9.16 \%$ de glicerol) para mejor conservación de los valores nutricionales, debido a que, a ese nivel de glicerol, aumenta la concentración de sodio en el músculo longissimus lumborum de corderos.

Por otra parte, Anneke et al. (2019), sin mencionar la pureza, constataron que una dieta de hasta $10 \%$ de glicerina bruta resulta en un alto depósito de grasa en la canal y músculo, derivando a un menor valor de fuerza de corte de la carne. Así, el uso de la glicerina bruta debe ser decidido previa evaluación económica de los demás componentes de la dieta (Socreppa et al., 2015), siendo posible reducir el costo de producción en bovinos de carne cuando el precio de ese ingrediente represente hasta $70 \%$ del precio del maíz (Strada et al., 2015).

\section{Conclusiones}

- Los trabajos incluidos en el metaanálisis evaluaron el efecto de la glicerina bruta en la dieta sin considerar al nivel de glicerol como efecto principal. 
Cuadro 5. Valores promedios de las características de la canal de cabras con diferentes niveles de inclusión de glicerol en la dieta en base a la materia seca

\begin{tabular}{lcccccc}
\hline \multirow{2}{*}{ Variables $^{1}$} & \multicolumn{3}{c}{ Niveles de glicerol $(\% \mathrm{MS})$} & \multirow{2}{*}{ CV $(\%)$} & \multicolumn{2}{c}{ valor $\mathrm{p}$} \\
\cline { 2 - 4 } \cline { 6 - 7 } & 0 & $1.27-4.38$ & $6.40-9.60$ & & Gli & $\mathrm{L}$ \\
\hline PCC $(\mathrm{kg})$ & 12.64 & 14.13 & 12.33 & 20.85 & 0.668 & 0.756 \\
PCF $(\mathrm{kg})$ & 12.30 & 13.87 & 12.10 & 20.41 & 0.609 & 0.789 \\
RCF $(\%)$ & 44.76 & 48.87 & 42.12 & 12.61 & 0.812 & 0.369 \\
AOL $\left(\mathrm{cm}^{2}\right)$ & 10.85 & 11.69 & 10.76 & 11.39 & 0.642 & 0.680 \\
\hline
\end{tabular}

$\mathrm{PCC}=$ peso de la canal caliente; $\mathrm{PCF}=$ peso de la canal fría; $\mathrm{RCF}=$ rendimiento de la canal fría; $\mathrm{AOL}=$ área de ojo de lomo; $\mathrm{CV}=$ coeficiente de variación

$\mathrm{Gli}=0 \%$ de glicerol vs. 1.27 a $9.60 \%$ de glicerol en la dieta

$\mathrm{L}=$ regresión lineal

${ }^{1}$ Cinco artículos incluidos

Cuadro 6. Valores promedios de los cortes comerciales y calidad de la carne de cabras con diferentes niveles de inclusión de glicerol en la dieta en base a la materia seca

\begin{tabular}{lcccccc}
\hline \multirow{2}{*}{ Variables } & \multicolumn{3}{c}{ Niveles de glicerol $(\% \mathrm{MS})$} & \multirow{2}{*}{ CV $(\%)$} & \multicolumn{2}{c}{ valor $\mathrm{p}$} \\
\cline { 2 - 4 } \cline { 7 - 7 } & 0 & $1.27-4.38$ & $6.40-9.60$ & & Gli & $\mathrm{L}$ \\
\hline Pierna $(\mathrm{kg})^{1}$ & 1877 & 1906 & 1.823 & 20.07 & 0.993 & 0.840 \\
Lomo $(\mathrm{kg})^{2}$ & 0.818 & 0.822 & 0.808 & 20.20 & 0.987 & 0.935 \\
Paleta $(\mathrm{kg})^{1}$ & 1486 & 1501 & 1455 & 13.36 & 0.963 & 0.800 \\
$\mathrm{pH}(24 \mathrm{~h})^{3}$ & 6.05 & 6.58 & 5.90 & 6.65 & 0.651 & 0.285 \\
FC $\left(\mathrm{kgf} / \mathrm{cm}^{2}\right)^{2}$ & 4.02 & 3.43 & 3.40 & 20.79 & 0.189 & 0.310 \\
Humedad $(\%)^{2}$ & 73.28 & 73.64 & 73.16 & 0.98 & 0.735 & 0.678 \\
Mineral $(\%)^{2}$ & 1.42 & 1.49 & 1.26 & 17.78 & 0.940 & 0.885 \\
\hline
\end{tabular}

$\mathrm{FC}=$ fuerza de cizallamiento; $\mathrm{CV}=$ coeficiente de variación

$\mathrm{Gli}=0 \%$ de glicerol vs. 1.27 a $9.60 \%$ de glicerol en la dieta

$\mathrm{L}=$ regresión lineal

${ }^{1}$ Cinco artículos incluidos; ${ }^{2}$ Cuatro artículos incluidos; ${ }^{3}$ Tres artículos incluidos 
- Los estudios con caprinos en engorde utilizando glicerol como fuente energética demostraron resultados semejantes a los tratamientos testigos ( sin glicerol en la dieta), sin encontrar un efecto limitante para su uso en concentraciones de hasta $9.60 \%$, en relación al comportamiento productivo, digestibilidad de los nutrientes y características de la canal y carne.

\section{Literatura Citada}

1. AbuGhazaleh AA, Abo El-Nor S, Ibrahim SA. 2011. The effect of replacing corn with glycerol on ruminal bacteria in continuous culture fermenters. J Anim Physiol An N 95: 313 319. doi: 10.1111/j.1439-0396.2010.01056.x

2. Alves e Dias C, Vieira e Silva F, Matos AM, Oliveira LLS, Oliveira AMF, Rocha Júnior VR, Batista LF, et al. 2018. Performance and carcass characteristics of goats fed crude glycerin in the feedlot and during pre slaughter lairage. Rev Bras Zootecn 47: e20170191. doi: 10.1590/rbz4720170191

3. Almeida MTC, Ezequiel JMB, Paschoaloto JR, Perez HL, Carvalho $V B$, Castro Filho ES, van Cleef EHCB. 2017. Effects of high concentrations of crude glycerin in diets for feedlot lambs: feeding behaviour, growth performance, carcass and non-carcass traits. Anim Prod Sci 5: 1271-1278. doi: 10.1071/AN16628

4. Anneke, Wattanachant C, Wattanachant S. 2019. Effects of supplementing crude glycerin in concentrated diet and castration on carcass characteristics and meat quality of Thai Native x Anglo Nubian goats. Walailak J Sci Technol 16: 477-486. doi: 10.48048/wjst.2019.3355

5. [ANP] Agencia Nacional de Petróleo. 2019. Rio de Janeiro: Anuário estatístico 2019. [Internet]. Disponible en: http:// www.anp.gov.br/publicacoes/anuarioestatistico/5237-anuario-estatistico-2019
6. Araújo LS, Marques CAT, Bezerra LR, Araújo MJ, Freitas NE, Santos CB, Torreão JNC, et al. 2019. Can crude glycerin and environmental conditions of semiarid affect the biological rhythm of ingestive behavior and physiological parameters of lactating goats? Biol Rhythm Res 52: 50-59. doi: 10.1080/ 09291016.2019.1583504

7. Avila JS, Chaves AV, Hernandez-Calva M, Beauchemin KA, McGinn SM, Wang Y, Harstad OM, et al. 2011. Effects of replacing barley grain in feedlot diets with increasing levels of glycerol on in vitro fermentation and methane production. Anim Feed Sci Tech 166-167: 265-268. doi: 10.1016/j.anifeedsci.2011.04.016

8. Barros MCC, Marques JA, Silva FF, Silva RR, Guimarães GS, Silva LL, Araújo FL. 2105. Glicerina bruta na dieta de ovinos confinados: consumo, digestibilidade, desempenho, medidas morfométricas da carcaça e características da carne. Semin-Cienc Agrar 36: 453-466. doi: 10.5433/1679-0359.2015v36n1p453

9. Bezerra H, Santos E, Oliveira J, Carvalho G, Silva F, Cassuce M, Perzzo A, Zanine A, Pinho R. 2019. Performance and ruminal parameters of Boer crossbred goats fed diets that contain crude glycerin. Animals 9: 967. doi: 10.3390/ani9110967

10. Castagnino PS, Fiorentini G, Dallantonia EE, San Vito E, Messana JD, Torrecilhas JA, Silva Sobrinho $A G S$, et al. 2018. Fatty acid profile and carcass traits of feedlot Nellore cattle fed crude glycerin and virginiamycin. Meat Sci 140: 51-58. doi: 10.1016/ j.meatsci.2018.02.013

11. Chanjula P, Cherdthong A. 2018. Effects of crude glycerin from waste vegetable oil in diets on performance and carcass characteristics of feedlot goats. Asian Australas J Anim 31: 514-521. doi: 10.5713/ajas. 17.0194 
12. Chanjula P. 2018. Use of crude glycerin as an energy source for goat diets: a review. Dairy Vet Sci J 2: 555578. doi: 10.19080/JDVS.2017.02.555578

13. Chanjula P, Pongprayoon S, Kongpan S, Cherdthong A. 2016. Effects of crude glycerin from waste vegetable oil supplementation on feed intake, ruminal fermentation characteristics, and nitrogen utilization of goats. Trop Anim Health Pro 48: 995-1004. doi: 10.1007/ s11250-016-1047-0

14. Chanjula P, Pakdeechanuan P, Wattanasit S. 2015. Effects of feeding crude glycerin on feedlot performance andcarcass characteristics in finishing goats. Small Ruminant Res 123: 95-102. doi: 10.1016/j.smallrumres.2014.11.011

15. Chanjula P, Pakdeechanuan P, Wattanasit S. 2014. Effects of dietary crude glycerin supplementation on nutrient digestibility, ruminal fermentation, blood metabolites, and nitrogen balance of goats. Asian Australas J Anim 27: 365-374. doi: 10.5713/ajas.2013.13494

16. Dias JC, Silveira ALF, Lançanova JAC, Hill JAG, Moletta JL. 2016. Crude glycerin in meat goat diets: intake, performance and carcass traits. Cienc Rural 46: 719-724. doi: 10.1590/0103$8478 \mathrm{cr} 20141489$

17. Gomes RN, Paula TA, Carvalho FFR, Ferreira MA, Barreto LMG, Neves MLMW, Mendes GO, et al. 2020a. The effect of crude glycerin of low purity replacing corn on goats' diets in feedlot in semiarid áreas. J Adv Vet Res 10: 66-72.

18. Gomes RN, Paula TA, Carvalho FFR, Cordeiro EHA, Nascimento HFA, Mendes GO, Oliveira AB, et al. $2020 b$. Low-purity crude glycerin as a nutrient substitute for corn: the effect on yields of goat organs and viscera. Acta Sci 42: e48650. doi: 10.4025/actascianimsci.v42i1.48650

19. Kholif $\boldsymbol{A E}$. 2019. Glycerol use in dairy diets: A systemic review. Anim Nutr 5: 209e216. doi: 10.1016/j.aninu.2019.-06.002
20. Lei $N^{o} 11.097 / 2005$. Dispõe sobre a introdução do biodiesel na matriz energética brasileira. Brasília, 13 de janeiro de 2005. [Internet]. Disponible en: https:/ /www.planalto.gov.br/ccivil_03/ ato2004-2006/2005/lei/111097.htm

21. Ley $N^{o}$ 2.748/2005. Fomento de los biocombustibles. Asunción, 07 de octubre de 2005. [Internet]. Disponible en: https://www.bacn.gov.py/leyesparaguayas $/ 2537 /$ fomento-de-losbiocombustibles

22. Machado SM, Sales ECJ, Rigueira JPS, Pires DAA, Silva AF, Monção FP. 2019. Glicerina bruta melhora o valor nutricional de silagem de milheto colhido em duas idades. Agrarian 12: 204-213. doi: 10.30612/agrarian.v12i44.8893

23. Matos AM, Silva FV, Oliveira LLS, Borges I, Ruas JRM, Novaes CG, Novais FC, Alves DD, Oliveira AMF. 2018. Strategies for the supply of crude glycerin for kids in pre-slaughter lairage. Small Ruminant Res 167: 87-91. doi: 10.1016/j.smallrumres.2018.08.010

24. Moher D, Liberati A, Tetzlaff J, Altman DG, The Prisma Group. 2009. Preferred reporting items for systematic reviews and meta-analyses: the PRISMA statement. Plos Med 6: e1000097. https://doi.org/10.1371/journal.pmed.1000097

25. Novais-Eiras D, Pinto de Carvalho GG, Leite LC, Eiras CE, Freitas Junior JE, Santos Pina D, Ferreira FG, et al. 2018. Crude glycerin in the feed supplementation of lactating goats on pasture. Small Ruminant Res 168: 3946. doi: 10.1016/j.smallrumres.2018.09.001

26. Oliveira AMF, Vieira e Silva F, Oliveira LLS, Rocha Júnior VR, Novaes CG, Novais FC, Rigueira JPS. 2020. Mineral content of longissimus lumborum from growing lambs fed crude glycerin. Meat Sci 169: 108222. doi: 10.1016/j.meatsci.2020.108222 
27. Paiva PG, Del Valle TA, Jesus EF, Bettero VP, Almeida GF, Bueno ICS, Bradford BJ, Rennó FP. 2016. Effects of crude glycerin on milk composition, nutrient digestibility and ruminal fermentation of dairy cows fed corn silage-based diets. Anim Feed Sci Tech 212: 136-142. doi: 10.1016/j.anifeedsci.2015.12.016

28. Pinto ABF, Naves LP, Lima IG, Garbossa CAP, Silva Júnior SR, Barbosa AMS, Maluf CL, et al. 2019. Metabolism of glycerol in pigs fed diets containing mixed crude glycerin and badrenergic agonist. Anim Prod Sci 59: 1631-1639. doi: 10.1071/AN18210

29. Rocha KS, Parente HN, Parente MOM, Ferreira EM, Araújo JS, Queiroga RCRE, Madruga MS, et al. 2015. Fatty acid profile, chemical composition, and sensory effects of crude glycerin on the longissimus dorsi of crossbred Boer goat kids. Rev Bras Zootecn 44: 263-268. doi: 10.1590/s180692902015000700005

30. Santos DA, Parente HN, Parente MOM, Rocha KS, Ferreira EM, Alves $A \boldsymbol{A}$. 2015. Desempenho produtivo de cabritos alimentados com glicerina bruta. Cienc Rural 45: 690-696. doi: 10.1590/ 0103-8478cr20140536

31. Schröder A, Südekum KH. 2007. Glycerol as a by-product of biodiesel production in diets for ruminants. University of Kiel, Kiel, Germany. [Internet]. Available in: https://dairycattle.extension.org/glycerol-as-a-feedingredient-in-dairy-rations/

32. Silva MC, Vaz RGMV, Rodrigues KF, Stringhini JH, Sousa LF, Fonseca FLR, Augusto WF, et al. 2019. Purified glycerin in balanced diets of broiler chickens treated from 1 to 42 days of age. Rev Bras Zootecn 48: e20180205. doi: 10.1590/rbz4820180205

33. Socreppa LM, Moraes EHBK, Moraes KAK, Oliveira AS, Drosghic LCAB, Botini LA, Stinguel H. 2015. Glicerina bruta para bovinos de corte em pastejo no período das águas: viabilidade produtiva e económica. Rev Bras Saúde Prod Anim 16:232-243.

34. Strada ESO, Silva RR, Carvalho GGP, Barbosa LP, Araújo FL, Leite MCP, Leite LC, et al. 2015. Glicerina de baixa pureza na suplementação de bovinos terminados a pasto: análise bioeconômica. Semin-Cienc Agrar 36: 2195-2210. doi: 10.5433/1679-0359.2015v36n3 Supl1p2195

35. Theisen MT, Bombardelli RA, Meurer F, Ferreira RL, Silva LCR. 2019. Crude glycerol inclusion in diets for postlarvae Rhamdia voulezi and Rhamdia branneri. Aquac Res 51: 1313-1316. doi: 10.1111/are.14465

36. Valença RL, Silva Sobrinho AGS, Romanzini EP, Andrade N, Borghi TH, Zeola NMBL, Cirne LGA, Oliveira VS. 2020. Peanut meal and crude glycerin in lamb diets: meat quality and fatty acid profile. Small Ruminant Res 185: 106076. doi: 10.1016/j.smallrumres.2020.106076

37. van Cleef EHCB, D'Áurea AP, Fávaro $V R$, van Cleef FOS, Barducci RS, Almeida MTC, Machado Neto OR, Ezequiel JMB. 2017. Effects of dietary inclusion of high concentrations of crude glycerin on meat quality and fatty acid profile of feedlot fed Nellore Bulls. Plos One 12: e0179830. doi: 10.1371/ journal.pone. 0179830 